\title{
Effect of Etching Time on Optical and Thermal Properties of p-Type Porous Silicon Prepared by Electrical Anodisation Method
}

\author{
Kasra Behzad, Wan Mahmood Mat Yunus, Zainal Abidin Talib, Azmi Zakaria, \\ Afarin Bahrami, and Esmaeil Shahriari
}

Department of Physics, Faculty of Science, Universiti Putra Malaysia, Selangor, 43400 Serdang, Malaysia

Correspondence should be addressed to Wan Mahmood Mat Yunus, mahmood@science.upm.edu.my

Received 23 August 2011; Revised 12 October 2011; Accepted 26 October 2011

Academic Editor: Ci-Ling Pan

Copyright ( $) 2012$ Kasra Behzad et al. This is an open access article distributed under the Creative Commons Attribution License, which permits unrestricted use, distribution, and reproduction in any medium, provided the original work is properly cited.

\begin{abstract}
The porous silicon (PSi) layers were formed on p-type silicon ( $\mathrm{Si}$ ) wafer. The six samples were anodised electrically with $30 \mathrm{~mA} / \mathrm{cm}^{2}$ fixed current density for different etching times. The structural, optical, and thermal properties of porous silicon on silicon substrates were investigated by photoluminescence (PL), photoacoustic spectroscopy (PAS), and UV-Vis-NIR spectrophotometer. The thickness and porosity of the layers were measured using the gravimetric method. The band gap of the samples was measured through the photoluminescence (PL) peak and absorption spectra, then they were compared. It shows that band gap value increases by raising the porosity. Photoacoustic spectroscopy (PAS) was carried out for measuring the thermal diffusivity (TD) of the samples.
\end{abstract}

\section{Introduction}

When crystalline silicon (c-Si) wafers are electrochemically etched in hydrofluoric acid (HF) at specific current densities, pores are formed, which is known as a porous silicon (PSi) layer [1]. This is an interesting material due to its unique and unusual optical and electrical properties compared to bulk Si substrate. Structurally, PSi is very complicated [2]. Some published papers indicate that PSi layers consist of Si columns and pores or isolated nanocrystallites [3]. On the other hand, PSi may be considered as a system of interconnected quantum wells, the so-called quantum sponge [4]. Nevertheless, the properties of PSi, such as porosity, thickness, pore diameter and, microstructure of silicon, have been reported to depend on anodisation conditions, including the electrolyte, current density, wafer type and resistivity, etching time, and temperature [5]. The first report of room temperature visible photoluminescence (PL) from porous silicon (PSi) structures has attracted wide interest in the scientific community [6]. The mechanism of light emission in porous silicon is not fully understood. One popular hypothesis is that luminescence is due to quantum confinement of charge carriers in narrow crystalline silicon walls separating the pores [6]. The increase in the band gap of PSi is possible by reducing the size of the nanocrystallites [7]. Another hypothesis asserts the existence of luminescent surface species trapped on the inner pore walls as the source of light emission [8]. Another concludes that the origin of luminescence can be traced to the presence of surface-confined molecular emitters, such as siloxene $\left(\mathrm{Si}_{6} \mathrm{O}_{3} \mathrm{H}_{6}\right)$ derivative, present in porous silicon [9]. It also has been indicated that the presence of oxygen shallow donors of binding energy in the $0.1 \mathrm{eV}$ range shows a distinct correlation with the intensity of the red PL observed in porous silicon [10]. Another competing model for the source of the luminescence is the nonbridging oxygen hole centres (NBOHCs) [11]. Another study suggesting a surface bound emitter as the source of the PSi photoluminescence, as indicated by the observed features in the form of the fluorophors associated with the silicon oxyhydrides, has been presented [12]. All the hypotheses are based on the unique area microstructure of PSi with large surface area, which is characterised by high porosity and crystalline structure with typical dimensions on the order of nanometers to a few micrometers [13]. Porous silicon has 
considerable attention for solar cell and sensor [14] applications. Its luminescence properties, large surface area, and compatibility with silicon-based devices are good reasons that cause the PSi to be used in the sensors and the solar cells. Related to these applications, the ability of adjustment and control of some parameters are more important. The band gap and the thermal diffusivity are two important parameters in sensors, solar cells $[15,16]$, electronic [17] and optoelectronic $[18,19]$ devices, thermal flow sensors [20], isolators [21], and fuel cells [22,23]. In this study, we will study on the band gap and the thermal diffusivity of PSi samples prepared by electrical anodisation method in different etching time. The structural properties also were studied by measuring the thickness and the porosity; the optical properties were studied by PL and UV-Vis-NIR spectroscopy, then the extracted values for band gap by these two methods were compared finally. The thermal diffusivity (TD) was measured by PAS method. Similar researches were done before but in this study the band gap values of the two methods will be measured and compared. This point make this study distinct from previous studies.

\section{Materials and Methods}

2.1. Sample Preparation. All the samples were formed on $\left(\begin{array}{lll}1 & 0 & 0\end{array}\right)$ p-type silicon single crystal wafers of $520 \mu \mathrm{m}$ and resistivity $1-10 \mathrm{ohm} \cdot \mathrm{cm}$ polished on one side. The wafers were cut into rectangular pieces. The typical area of the pieces is approximately $5 \mathrm{~cm}^{2}$. In order to provide a uniform current distribution across the surface, an aluminium layer was deposited on the back side of all samples. Each sample was placed in the bottom of cylindrical Teflon made cell and fixed by an aluminium plate as backing material. The cell has two electrodes a $\mathrm{p}$-Si wafer as anode and platinum rod as a cathode that was placed perpendicular to the anode by a distance of $1 \mathrm{~cm}$. The samples were obtained by varying the time duration of the etching process at a constant concentration of $48-50 \% \mathrm{HF}, 99.90 \%$ ethanol and distilled water solution $\left(\mathrm{HF}: \mathrm{C}_{2} \mathrm{H}_{5} \mathrm{OH}: \mathrm{H}_{2} \mathrm{O}\right)$ taken in the volume ratio of $1: 2: 1$. The electric current was supplied using an ADCMT 6243 , DC current source. The samples in this study were prepared under $30 \mathrm{~mA} / \mathrm{cm}^{2}$ current density in 10, 20, 30, 40, 50 , and 60 min anodisation time that are denoted as sample $1,2,3,4,5$, and 6 , respectively.

2.2. Characterisations. Thickness and porosity of the samples were calculated by the gravimetric method [18]. The samples are weighted before anodisation $\left(m_{1}\right)$, just after anodisation $\left(m_{2}\right)$, and after dissolution of the porous silicon layer in a molar $\mathrm{NaOH}$ aqueous solution $\left(m_{3}\right)$. The porosity and thickness are given by the following equations, respectively,

$$
\begin{gathered}
P(\%)=\frac{m_{1}-m_{2}}{m_{1}-m_{3}} \times 100, \\
d=\frac{m_{1}-m_{3}}{\rho S},
\end{gathered}
$$

where $\rho$ is the Si density and $S$ the anodised surface. The thickness of the PSi layers also was rechecked by Ambios Technology, stylus profilometer, XP-200.

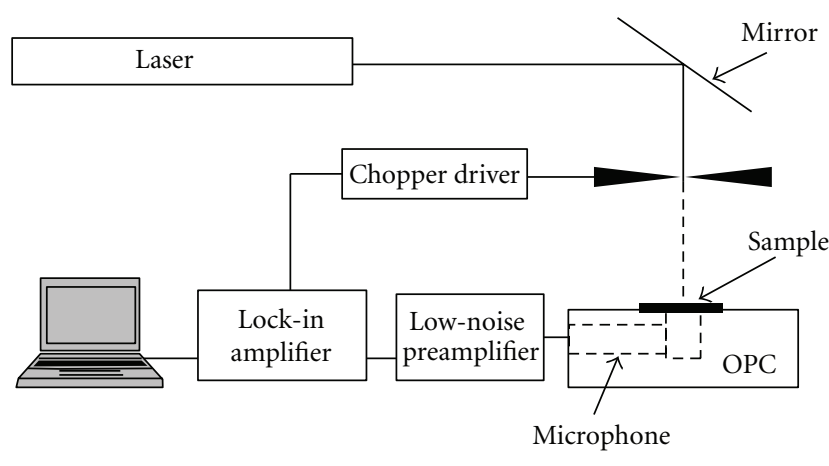

Figure 1: Schematic diagram of PAS setup.

PSi-based structures have been reported to luminescence in the NIR, visible and near UV. Based on our conditions, the results are in the visible field so-called S-band [24]. The S-band can be tuned from close to the bulk Si band gap $(1.14 \mathrm{eV})$ through the whole visible range. The S-band large spectral width comes from inhomogeneous broadening, and its spectral position depends on porosity [18]. By an incident of a light with sufficient energy on a material, photons are absorbed and excite the electrons to excitation levels. These electrons relax and return to the ground state. By radiative relaxation, the emitted beam is called PL. The PL spectrum provides the transition energies, which can be used to determine the band gap. PL intensity also related to the rates of radiative and nonradiative recombination rates [25]. Photoluminescence spectroscopy was performed by a Power Technology diode laser, having an excitation wavelength of $405 \mathrm{~nm}$. The luminescence emitted is analyzed by an Ocean Optics high-resolution spectrometer, HR4000. The excited and emitted beams pass through an Ocean Optics bifurcated optical fibre.

All the PAS setups consist of three parts: light source, detector, and data analysing system. A Melles Griot $\mathrm{HeNe}$ laser of $632.8 \mathrm{~nm}$ at power of $75 \mathrm{~mW}$ was used as a light source that was modulated by Stanford Research Systems optical chopper SR540; a handmade open photoacoustic cell (OPC) was used as a detector. A Stanford Research Systems low-noise preamplifier, SR560, amplified the very weak output signal from OPC and sent it to a Stanford Research Systems lock-in amplifier SR530. A program written in Lab VIEW was controlled and collected the data from the lock-in amplifier via a GPIB bus, Figure 1. The PA signal was obtained by chopping frequency in $14-205 \mathrm{~Hz}$.

To describe the result of PAS, we refer to the thermal piston as Rosencwaig and Gersho (RG) present it in the RG theory [26]. They believed that the source of the acoustic signal in the cell is caused by the periodic heat flow of the solid to the ambient gas as the solid heated by the modulated monochromatic beam. The pressure fluctuations are described as

$$
\delta p=\frac{\gamma p_{0} I_{0}\left(\alpha_{g} \alpha_{s}\right)^{1 / 2} \exp j(\omega t-\pi / 2)}{2 \pi T_{0} l_{g} \kappa_{s} f \sinh \left(l_{s} \sigma_{s}\right)},
$$


TABLE 1: Porosity and thickness of samples.

\begin{tabular}{lccccc}
\hline Sample no. & Substrate & Current density $\left(\mathrm{mA} / \mathrm{cm}^{2}\right)$ & Etching time $(\mathrm{min})$ & Porosity $(\%)$ & Thickness $(\mu \mathrm{m})$ \\
\hline 1 & $\mathrm{p}-\mathrm{Si}$ & 30 & 10 & 62.37 & 72.02 \\
2 & $\mathrm{p}-\mathrm{Si}$ & 30 & 20 & 76.72 & 22.84 \\
3 & $\mathrm{p}-\mathrm{Si}$ & 30 & 30 & 79.13 & 33.35 \\
4 & $\mathrm{p}-\mathrm{Si}$ & 30 & 40 & 79.68 & 45.00 \\
5 & $\mathrm{p}-\mathrm{Si}$ & 30 & 50 & 80.20 & 57.21 \\
6 & $\mathrm{p}-\mathrm{Si}$ & 30 & 60 & 69.20 \\
\hline
\end{tabular}

where $\gamma$ is the air specific-heat ratio, $P_{0}$ the ambient pressure, $I_{0}$ the incident light beam intensity, $f$ the chopping frequen$\mathrm{cy}$, and $l_{i}, k_{i}, \alpha_{i}$, are the thickness, thermal conductivity, and TD of material $i$, respectively. The subscript $i$ can take the $s$ and $g$ for the sample and gas, respectively. TD $\left(\alpha_{i}\right)$ is the rate of propagation a temperature pulse in a material between two points

$$
\alpha=\frac{k}{\rho C_{v}}
$$

where $\alpha_{i}$ is TD, $k$ is thermal conductivity $\rho$ is density, and $C_{v}$ is the specific heat of the sample. The complex thermal diffusion coefficient of material $i$ defined as [26]

$$
\sigma_{i}=(1+j) a_{i}, \quad a_{i}=\frac{1}{\mu_{i}}=\left(\frac{\pi f}{\alpha_{i}}\right)^{1 / 2},
$$

where $\mu_{i}$ is the thermal diffusion length. Particularly, for a thermally thick sample $\left(l_{s} \sigma_{s} \gg 1\right)$, PA amplitude $(S)$ varies as

$$
S=\frac{A}{f} \exp (-b \sqrt{f}), \quad b=\left(\frac{\pi l_{s}^{2}}{\alpha_{s}}\right)^{1 / 2} .
$$

For measuring the TD of samples $\left(\alpha_{s}\right)$, the PA signal is plotted versus chopping frequency. By fitting the PA signal to (6) in the thermally thick area, TD value can be derived from $b$ value as the $l_{s}$ is measured with a micrometer screw gauge. TD value also obtains from characteristic frequency $\left(f_{c}\right)$. The characteristic frequency is the modulation frequency when it passes from thermally thin to thermally thick regime, and the thermal diffusion length $(\mu)$ will be equal to sample thickness, that is, $f=f_{C}, \mu_{S}=l_{S}$ [27]

$$
l_{S}=\left(\frac{\alpha_{S}}{\pi f_{C}}\right)^{1 / 2}, \quad \alpha_{S}=\pi f_{C} l_{s}^{2} .
$$

The samples also were characterized by a Shimadzu, UVVis-NIR Spectrophotometer UV3600. Samples were placed in chamber one by one then studied in 220 to $2600 \mathrm{~nm}$ wavelength. For measuring the band gap, the absorbance was changed to absorption by considering the thickness of each sample. By using the Tauc relation $(\alpha h v)^{m}=\beta\left(h v-E_{g}\right)$ and extrapolating the data on $(\alpha h \nu)^{m}=0$, where $\alpha$ is the absorption and $\nu=c / \lambda$ is the frequency of the incident beam, the band gap value was found for each sample and also c-Si [28].
TABLE 2: Comparison of the measured values for the TD of samples.

\begin{tabular}{lccc}
\hline $\begin{array}{l}\text { Sample/Sample } \\
\text { number }\end{array}$ & Porosity $(\%)$ & $\begin{array}{c}\alpha\left(\mathrm{cm}^{2} / \mathrm{s}\right) \\
\text { Measured }\end{array}$ & $\begin{array}{c}\alpha\left(\mathrm{cm}^{2} / \mathrm{s}\right) \\
\text { Literature }\end{array}$ \\
\hline $\mathrm{Al}$ & - & 0.97 & $0.96-1.12$ \\
$\mathrm{Si}$ & - & 0.87 & $0.85-0.97$ \\
1 & 62.37 & 0.78 & - \\
2 & 72.02 & 0.69 & - \\
3 & 76.72 & 0.64 & - \\
4 & 79.13 & 0.58 & - \\
5 & 79.68 & 0.56 & - \\
6 & 80.20 & 0.48 & - \\
\hline
\end{tabular}

\section{Results}

3.1. Thickness and Porosity. By using (1) and (2) after measuring the mass of samples, density of substrate $(\rho)$, and anodized area $(S)$, porosity percentage and thickness of the layer were calculated. The variation of porosity and thickness is shown in Figure 2 and the numerical values are presented in Table 1.

It can be noted that porosity initially increases rapidly, but after attaining a certain value, which in the present case is around $80 \%$, it roughly levels off. This value is achieved for around 50 min etching time. The average thickness of PSi layers grows linearly by increasing the etching time. The thickness of these samples also was measured by stylus profilometer. The results are in a good agreement with the mentioned values in Table 1.

3.2. Photoluminescence (PL). The PL emission peaks were obtained from six samples with the photoluminescence spectroscopy setup that was explained before. The room temperature PL measurements of various samples anodised at different times are shown in Figure 3(a). For the samples that anodised in 10, 20, 30, 40, 50, and $60 \mathrm{~min}$, the PL peaks, related to the S-band emission, observed at wavelength 656.5, $645.3,629.0,615.7,604.7$, and $588.0 \mathrm{~nm}$, respectively. As we have studied earlier, the porosity is increased by increasing the etching time. So, clearly the silicon structure size on the surface decreases by increasing the anodisation time. It causes the peaks to shift towards the lower wavelength or higher energy as the anodisation time is growing up. That is in agreement with the particle in a box theory. The energy 


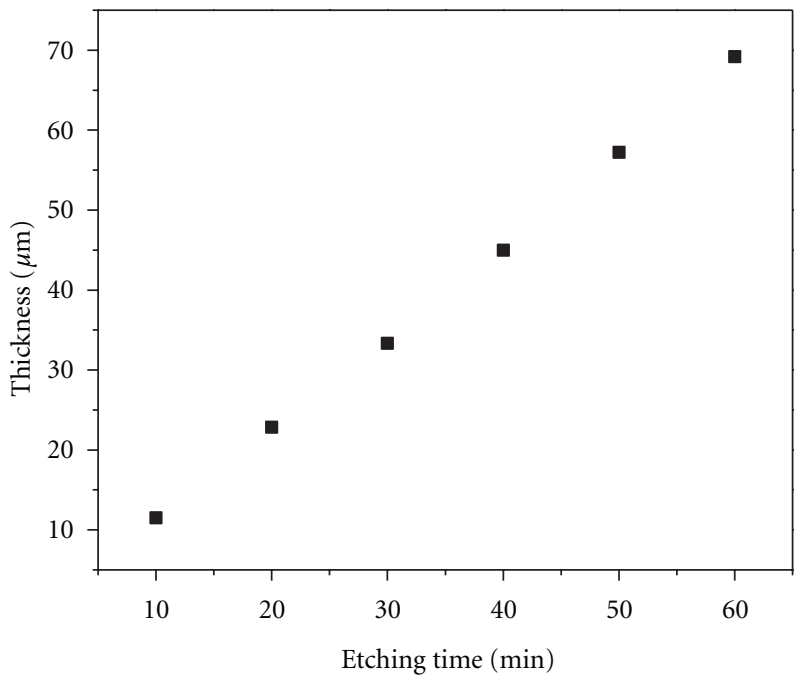

(a)

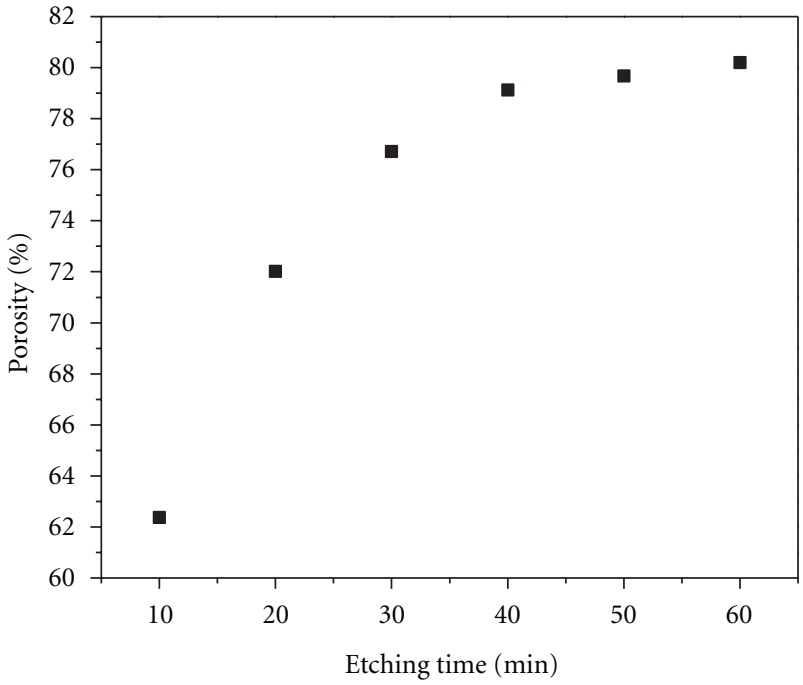

(b)

Figure 2: (a) Thickness as a function of etching time and (b) porosity as a function of etching time.

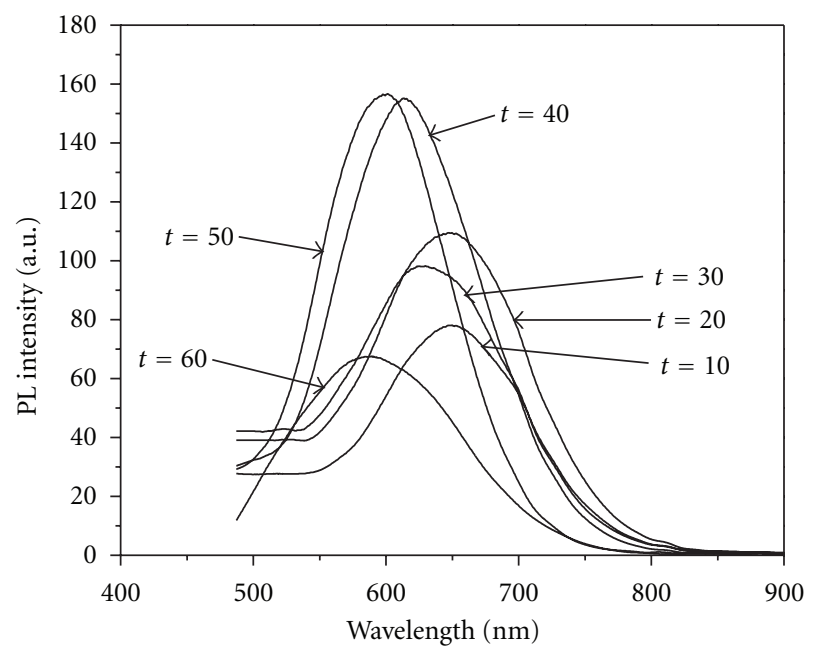

(a)

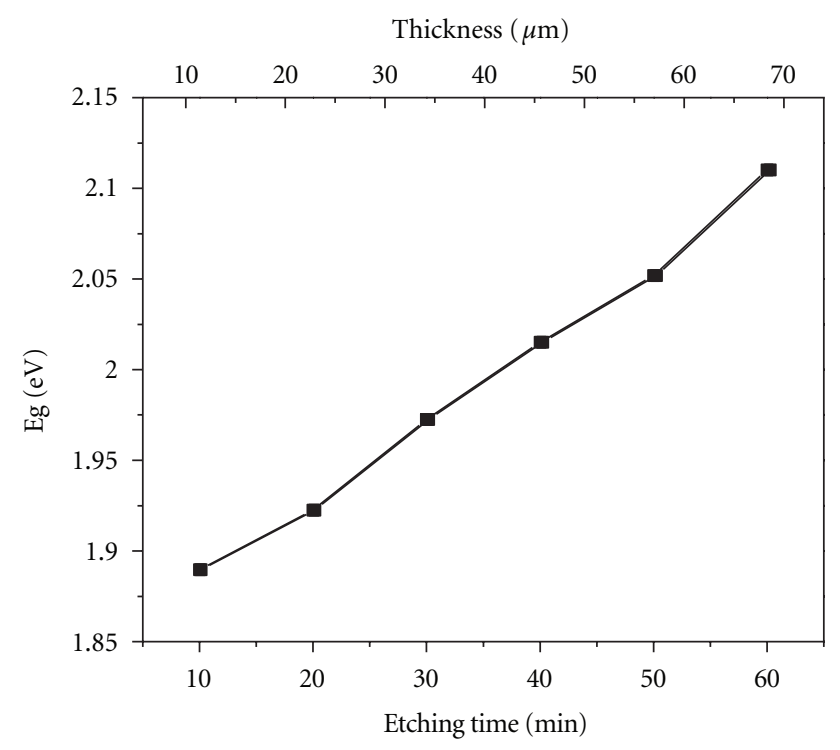

(b)

FIGURE 3: (a) PL peaks for different anodisation time excited by $405 \mathrm{~nm}$ and (b) band gap versus etching time/thickness.

gap versus anodisation time and PSi layer thickness are shown in Figure 3(b).

In this study, the position of the PL peak varies between $588.0-656.5 \mathrm{~nm}$ by changing the time from 10 to $60 \mathrm{~min}$. So the band gap of the PSi samples can be tuned in this interval by adjusting the time between $10-60 \mathrm{~min}$ at $30 \mathrm{~mA} / \mathrm{cm}^{2}$ current density for our conditions.

3.3. Photoacoustic Spectroscopy (PAS). Figure 4(a) shows the photoacoustic (PA) signal of the PSi/Si sample at modulation frequency between 14 and $205 \mathrm{~Hz}$. At low frequency, the thermal diffusion length is larger than the thickness of samples' so-called thermally thin regime. By increasing the frequency to a certain point, the thermal diffusion length will equal the sample thickness; this value of the frequency is named as characteristic frequency $\left(f_{c}\right)$. By increasing the frequency, it changes to the thermally thin regime [29]. So, the PA signals were fitted with (6) only in the thermally thick area as shown in Figure 4(b). The TD value also was calculated for all samples by finding the characteristic frequency using (7), and the values also confirmed by this method as well, Figure 4(c). 


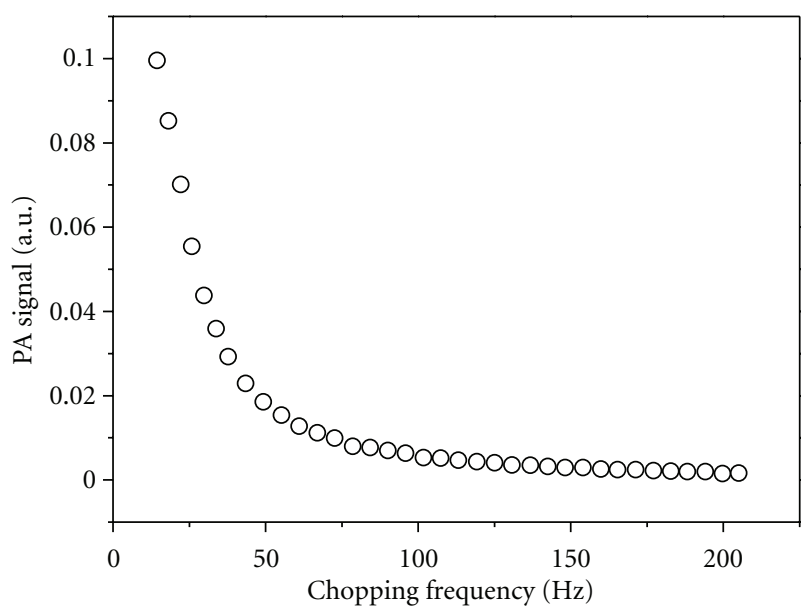

(a)

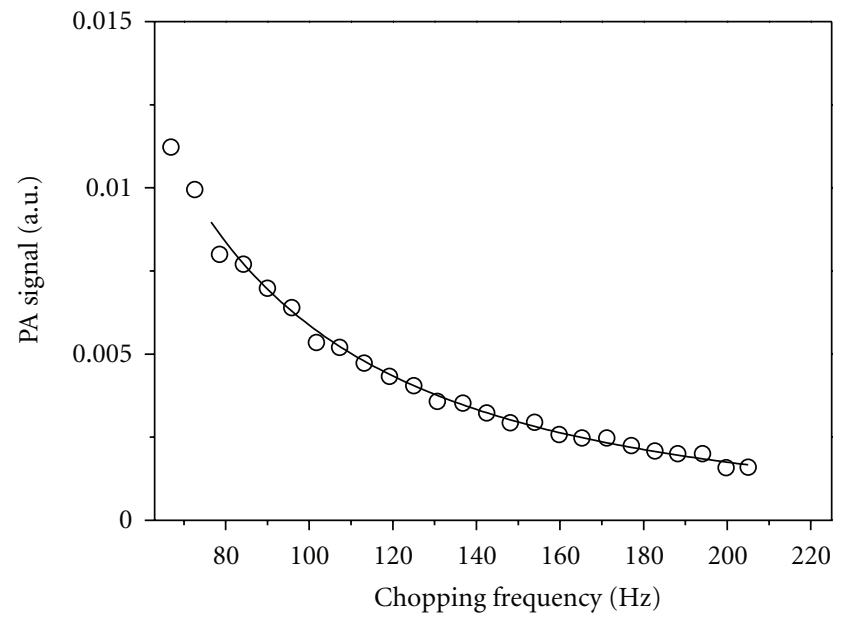

(b)

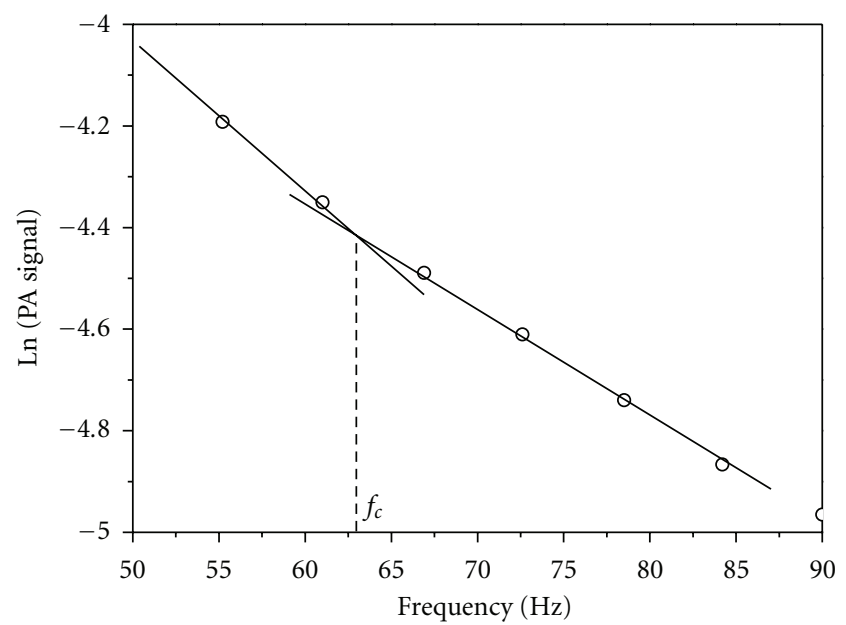

(c)

FIGURE 4: (a) PA spectra for PSi/Si sample under chopping frequency and (b) PA signal versus chopping frequency. The solid curve represents the best fit of the data to (6). (c) Ln (PA signal) versus chopping frequency for one of the samples.

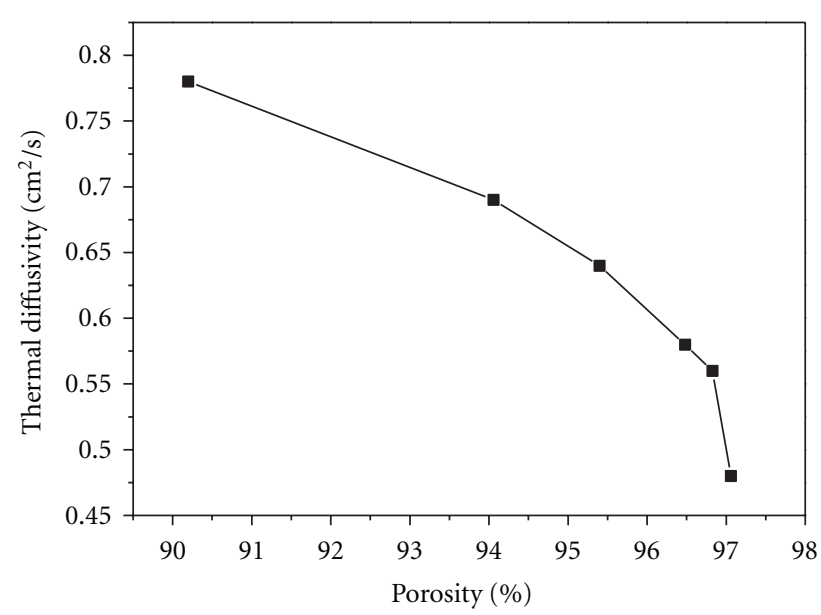

(a)

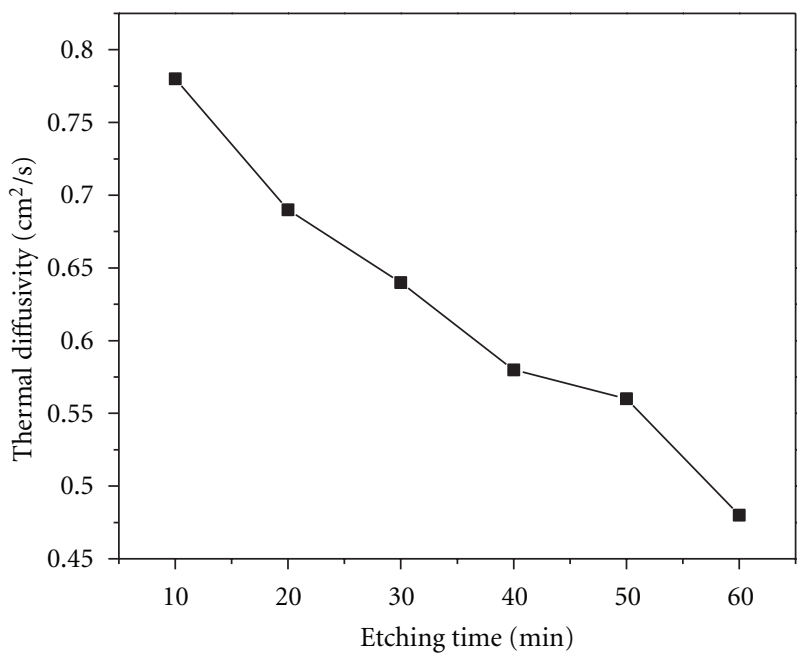

(b)

FIGURE 5: Variation of TD versus (a) porosity and (b) etching time for samples.

Before characterising the PSi samples, optical setup and measuring procedures were tested with high purity $\mathrm{Al}$ foil and $\mathrm{c}-\mathrm{Si}$ wafer and the thermal diffusivities have a good agreement with the literature reviews [30-33]. According to these literature reviews, these values are $\alpha_{\mathrm{Al}}=0.96-$ $1.12 \mathrm{~cm}^{2} / \mathrm{s}$ and $\alpha_{\mathrm{Si}}=0.85-0.97 \mathrm{~cm}^{2} / \mathrm{s}$ for $\mathrm{Al}$ and $\mathrm{Si}$, respectively.

The variations of the TD value of the samples are compared in Figure 5 and also represented in Table 2. These results confirm the good possibility for thermal insulation given by $\mathrm{PSi} / \mathrm{Si}$ structure in contrast to $\mathrm{c}-\mathrm{Si}$, which has higher TD. It can be useful in designing the silicon-based devices. Decreasing the TD by increasing the porosity is due to a decrease in the mean free path due to the phonon confinement in crystallite $[34,35]$. 


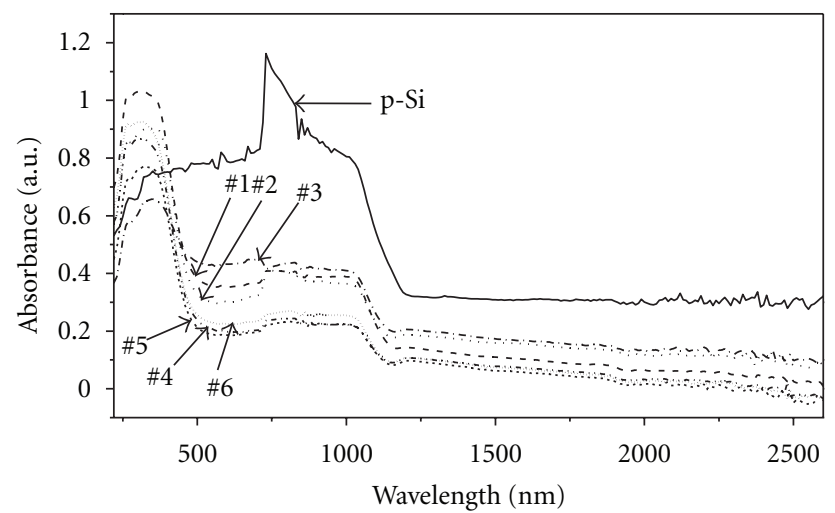

FIGURE 6: Diffuse absorbance spectra of p-Si and PSi samples.

TABle 3: Comparison of the Band gap values extract from Photoluminescence and UV-Vis-NIR spectroscopy.

\begin{tabular}{lccc}
\hline $\begin{array}{l}\text { Sample/Sample } \\
\text { number }\end{array}$ & Porosity $(\%)$ & \multicolumn{2}{c}{ Band gap (eV) } \\
& & PL & UV-Vis-NIR \\
\hline Si & - & 1.14 (as literature) & 1.09 \\
1 & 62.37 & 1.88 & 1.87 \\
2 & 72.02 & 1.92 & 1.89 \\
3 & 76.72 & 1.97 & 1.94 \\
4 & 79.13 & 2.01 & 1.98 \\
5 & 79.68 & 2.05 & 2.01 \\
6 & 80.20 & 2.11 & 2.09 \\
\hline
\end{tabular}

3.4. UV-Vis-NIR Spectroscopy. PSi/Si samples and c-Si wafer were used for the UV-Vis-NIR absorption studies represented in Figure 6. Two sets of slopes in this graph, in low and higher wavelengths, belong to PSi and Si, respectively.

Firstly, to establish the type of band-to-band transition in this study, the absorption data were fitted to equations for both indirect and direct band gap transitions. As per the literature reviews the band gap of the PSi samples are in 1.5$2.5 \mathrm{eV}$ range, so it has been taken into account $m=2$ for PSi samples. Figure 7 shows $(\alpha h \nu)^{2}$ versus energy plotted for the same data. Each graph was extrapolated on $(\alpha h \nu)^{2}=0$ to find the related band gap. Figure 8 shows it for one of the samples.

The calculated band gap of the samples by extrapolation of the absorption edge onto the energy axis and the extracted values from PL are reported in Table 3 and Figure 9. The average difference in band gap values that extracted from these two methods is around $1.8 \%$.

\section{Summary}

In summary, the samples were prepared by anodisation method under a fixed current density and six different etching times. We have studied the dependence of porous silicon thickness and porosity on etching time. The results show that

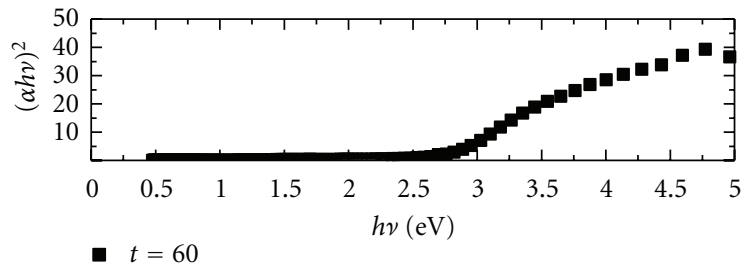

(a)

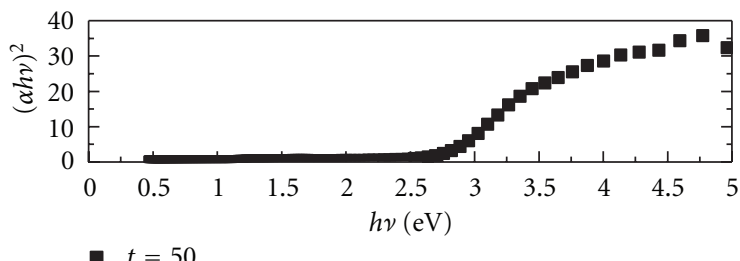

(b)

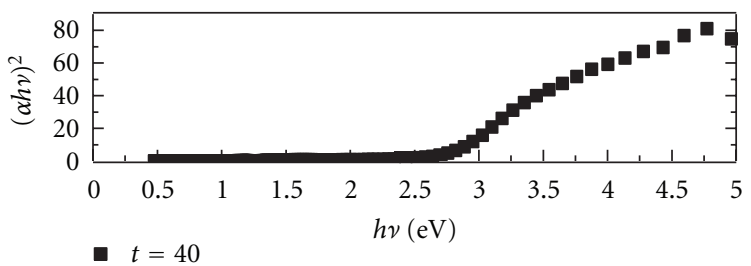

(c)

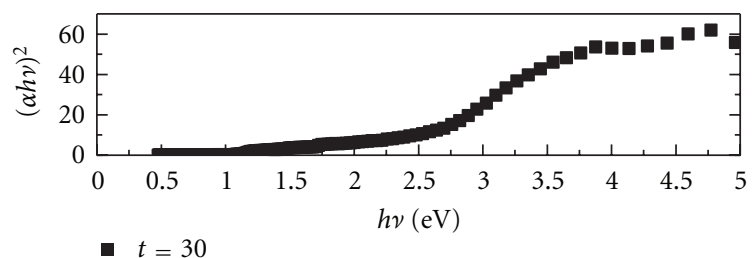

(d)

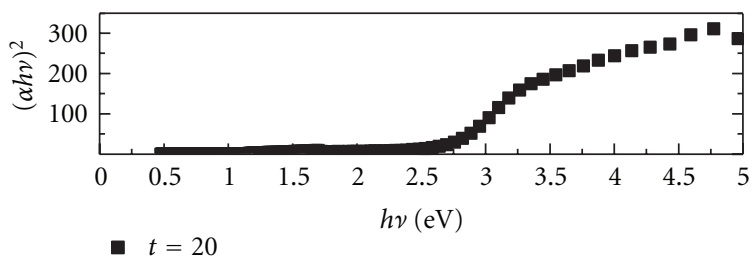

(e)

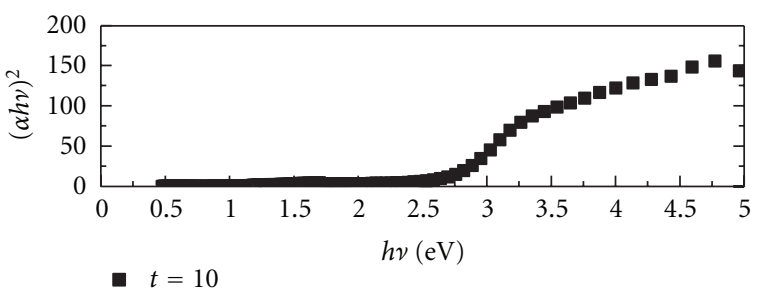

(f)

FIGURE 7: $(\alpha h v)^{2}$ versus energy for the PSi samples. 


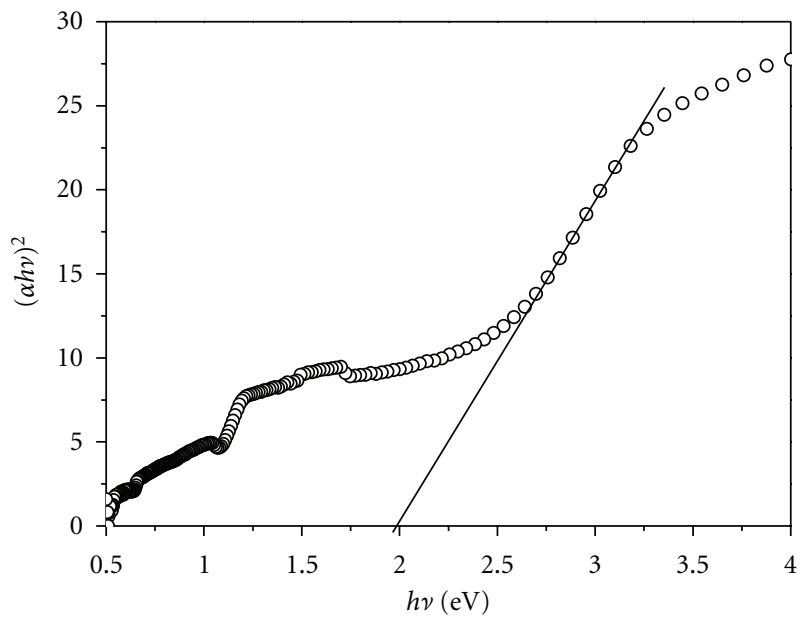

FIgURE 8: $(\alpha h v)^{2}$ versus $h v$ and its linear fitting line for a PSi sample.

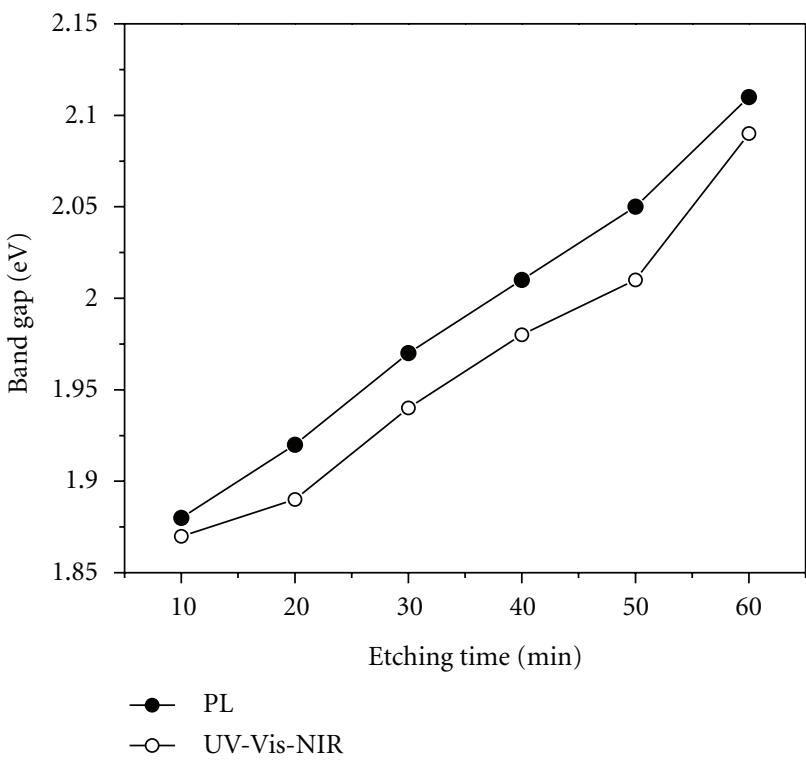

(a)

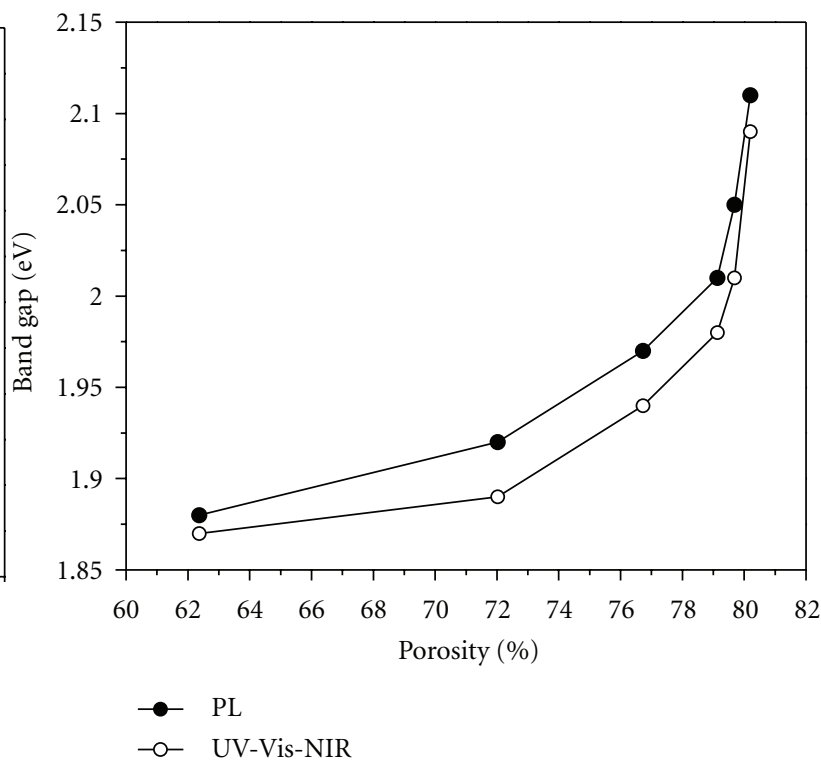

(b)

Figure 9: Band gap versus etching time (a) and porosity (b) extract from PL and UV-Vis-NIR spectroscopy.

the porosity increases until around $80 \%$ and then remains approximately constant, whereas the overall thickness of the porous silicon layer grows linearly in time. By increasing the porosity, the Si structures in PSi layer reduce. The samples were tuned optically and thermally by etching time. The PL result indicates a gradually blue shift in peaks with increasing the etching time that is due to changing the Si structure size in PSi layers. Furthermore, the samples were studied by UVVis-NIR spectroscopy and the band gap values of samples were extracted from this method also. By measuring the band gap through the PL and UV-Vis-NIR spectroscopies, it was found that the band gap value increases by etching time. Derived results for band gap from these two methods are in a good agreement with each other as described before. The results show that the band gap value can adjust by varying the etching time for different application. PAS was carried out to measure the TD. PAS results show that the TD was significantly smaller than that of the $\mathrm{Si}$, and decreased with increased porosity, that is due to a decrease in the mean free path due to the phonon confinement in crystallite. In different applications of the PSi, mentioned before, it needs to adjust the parameters in an optimum value, so they should be possible to tune. Depending on the application, the PSi samples should have different band gap or thermal diffusivity due to changing the etching time.

\section{Acknowledgments}

The authors gratefully acknowledge the Department of Physics, UPM for providing the research facilities to enable them 
to carry out this research. They would also like to acknowledge Ministry of Higher Education for the financial support through Fundamental Research Grant (01-11-08-664FR/ 5523664 and 01-04-10-861FR/5523901). Kasra Behzad also gratefully acknowledges Professor A. Mandelis and Assoc. Professor. Z. Gaburro for their helpful advices in this study.

\section{References}

[1] R. L. Smith and S. D. Collins, "Porous silicon formation mechanisms," Journal of Applied Physics, vol. 71, no. 8, pp. R1-R22, 1992.

[2] M. Balarin, O. Gamulin, M. Ivanda et al., "Structural, optical and electrical characterization of porous silicon prepared on thin silicon epitaxial layer," Journal of Molecular Structure, vol. 924-926, pp. 285-290, 2009.

[3] C. Lévy-Clément, S. Lust, M. Mamor, J. Rappich, and T. Dittrich, "Investigation of p-type macroporous silicon formation," Physica Status Solidi (A), vol. 202, no. 8, pp. 1390-1395, 2005.

[4] V. Lehmann and U. Gösele, "Porous silicon formation: a quantum wire effect," Applied Physics Letters, vol. 58, no. 8, pp. 856858, 1991.

[5] S. Z. You, Y. F. Long, Y. S. Xu et al., "Fabrication and characterization of thick porous silicon layers for rf circuits," Sensors and Actuators A, vol. 108, no. 1-3, pp. 117-120, 2003.

[6] L. T. Canham, "Silicon quantum wire array fabrication by electrochemical and chemical dissolution of wafers," Applied Physics Letters, vol. 57, no. 10, pp. 1046-1048, 1990.

[7] R. Behrensmeier, F. Namavar, G. B. Amisola, F. A. Otter, and J. M. Galligan, "Further evidence for quantum confinement in porous silicon," Applied Physics Letters, vol. 62, no. 19, pp. 2408-2410, 1993.

[8] F. Koch, V. Petrova-Koch, and T. Muschik, "The luminescence of porous Si: the case for the surface state mechanism," Journal of Luminescence, vol. 57, no. 1-6, pp. 271-281, 1993.

[9] M. S. Brandt, H. D. Fuchs, M. Stutzmann, J. Weber, and M. Cardona, "The origin of visible luminescencefrom "porous silicon": a new interpretation," Solid State Communications, vol. 81, no. 4, pp. 307-312, 1992.

[10] S. M. Prokes, W. E. Carlos, and O. J. Glembocki, "Defect-based model for room-temperature visible photoluminescence in porous silicon," Physical Review B, vol. 50, no. 23, pp. 1709317096, 1994.

[11] S. M. Prokes, "Light emission in thermally oxidized porous silicon: evidence for oxide-related luminescence," Applied Physics Letters, vol. 62, no. 25, pp. 3244-3246, 1993.

[12] J. L. Gole, F. P. Dudel, D. Grantier, and D. A. Dixon, "Origin of porous silicon photoluminescence: evidence for a surface bound oxyhydride-like emitter," Physical Review B, vol. 56, no. 4, pp. 2137-2153, 1997.

[13] K. B. Sundaram, J. Alizadeh, S. Albin, J. Zheng, and A. Lavarias, "Photoluminescence enhancement in porous silicon layers," Journal of Materials Science: Materials in Electronics, vol. 9, no. 4, pp. 271-274, 1998.

[14] S. Ozdemir and J. L. Gole, "The potential of porous silicon gas sensors," Current Opinion in Solid State and Materials Science, vol. 11, no. 5-6, pp. 92-100, 2007.

[15] L. C. Chen and B. H. Liu, "Porous silicon layer patterned from anodic aluminum oxide and application in $\mathrm{ZnPc}$ hybrid solar cell," Electrochemical and Solid-State Letters, vol. 13, no. 4, pp. H108-H111, 2010.
[16] N. Marrero, R. Guerrero-Lemus, B. González-Díaz, and D. Borchert, "Effect of porous silicon stain etched on large area alkaline textured crystalline silicon solar cells," Thin Solid Films, vol. 517, no. 8, pp. 2648-2650, 2009.

[17] Y. Qu, L. Liao, Y. Li, H. Zhang, Y. Huang, and X. Duan, "Electrically conductive and optically active porous silicon nanowires," Nano Letters, vol. 9, no. 12, pp. 4539-4543, 2009.

[18] O. Bisi, S. Ossicini, and L. Pavesi, "Porous silicon: a quantum sponge structure for silicon based optoelectronics," Surface Science Reports, vol. 38, no. 1-3, pp. 1-126, 2000.

[19] R. S. Dubey and D. K. Gautam, "Fabrication and characterization of porous silicon layers for applications in optoelectronics," Optical and Quantum Electronics, vol. 41, no. 3, pp. 189-201, 2009.

[20] G. Kaltsas, A. A. Nassiopoulos, and A. G. Nassiopoulou, "Characterization of a silicon thermal gas-flow sensor with porous silicon thermal isolation," IEEE Sensors Journal, vol. 2, no. 5, pp. 463-475, 2002.

[21] D. N. Pagonis, A. G. Nassiopoulou, and G. Kaltsas, "Porous silicon membranes over cavity for efficient local thermal isolation in Si thermal sensors," Journal of the Electrochemical Society, vol. 151, no. 8, pp. H174-H179, 2004.

[22] K. L. Chu, M. A. Shannon, and R. I. Masel, "Porous silicon fuel cells for micro power generation," Journal of Micromechanics and Microengineering, vol. 17, no. 9, pp. S243-S249, 2007.

[23] T. Pichonat and B. Gauthier-Manuel, "Realization of porous silicon based miniature fuel cells," Journal of Power Sources, vol. 154, no. 1, pp. 198-201, 2006.

[24] Z. Gaburro, N. Daldosso, and L. Pavesi, "Porous silicon," in Encyclopedia of Condensed Matter Physics, F. Bassani, J. Liedl, and P. Wyder, Eds., pp. 391-401, Elsevier, Oxford, UK, 2005.

[25] T. H. Gfroerer, "Photoluminescence in analysis of surfaces and interfaces," in Encyclopedia of Analytical Chemistry, John Wiley \& Sons, New York, NY, USA, 2006.

[26] A. Rosencwaig and A. Gersho, "Theory of the photoacoustic effect with solids," Journal of Applied Physics, vol. 47, no. 1, pp. 64-69, 1976.

[27] J. C. de Lima, M. Schmitt, T. A. Grandi et al., "Structural and photoacoustic studies of mechanically alloyed $\mathrm{Ga}_{40} \mathrm{Sb}_{38} \mathrm{Se}_{22}$ powder," Journal of Physics: Condensed Matter, vol. 19, no. 18, Article ID 186216, 2007.

[28] J. Tauc and A. Menth, "States in the gap," Journal of NonCrystalline Solids, vol. 8-10, pp. 569-585, 1972.

[29] E. Ramachandran, P. Raji, K. Ramachandran, and S. Natarajan, "Photoacoustic study of the thermal properties of calcium carbonate- the major constituent of pancreatic calculi," Crystal Research and Technology, vol. 41, no. 1, pp. 64-67, 2006.

[30] C. K. Sheng, W. M. M. Yunus, W. M. Z. W. Yunus, Z. A. Talib, and A. Kassim, "Characterization of thermal, optical and carrier transport properties of porous silicon using the photoacoustic technique," Physica B, vol. 403, no. 17, pp. 26342638, 2008.

[31] A. P. Neto, H. Vargas, N. F. Leite, and L. C. M. Miranda, "Photoacoustic characterization of semiconductors: transport properties and thermal diffusivity in GaAs and Si," Physical Review B, vol. 41, no. 14, pp. 9971-9979, 1990.

[32] Q. Shen and T. Toyoda, "Characterization of thermal properties of porous silicon film/silicon using photoacoustic technique," Journal of Thermal Analysis and Calorimetry, vol. 69, no. 3, pp. 1067-1073, 2002.

[33] A. Calderón, R. A. M. Hernández, S. A. Tomás, A. Cruz-Orea, and F. Sánchez Sinencio, "Method for measurement of the 
thermal diffusivity in solids: application to metals, semiconductors, and thin materials," Journal of Applied Physics, vol. 84, no. 11, pp. 6327-6329, 1998.

[34] R. Srinivasan, M. Jayachandran, and K. Ramachandran, "Photoacoustic studies on optical and thermal properties of p-type and n-type nanostructured porous silicon for (100) and (111) orientations," Crystal Research and Technology, vol. 42, no. 3, pp. 266-274, 2007.

[35] J. Zou and A. Balandin, "Phonon heat conduction in a semiconductor nanowire," Journal of Applied Physics, vol. 89, no. 5, pp. 2932-2938, 2001. 

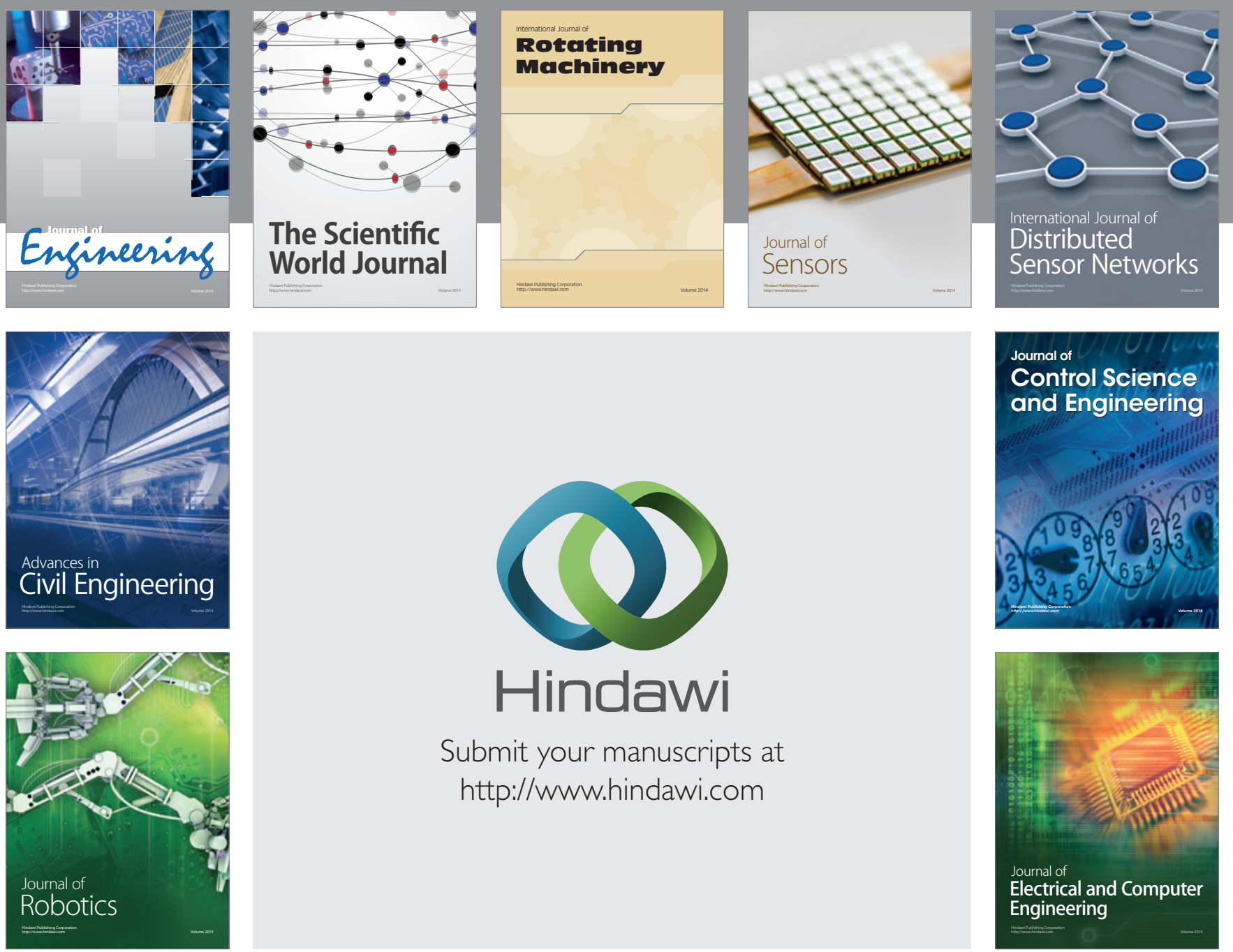

Submit your manuscripts at

http://www.hindawi.com
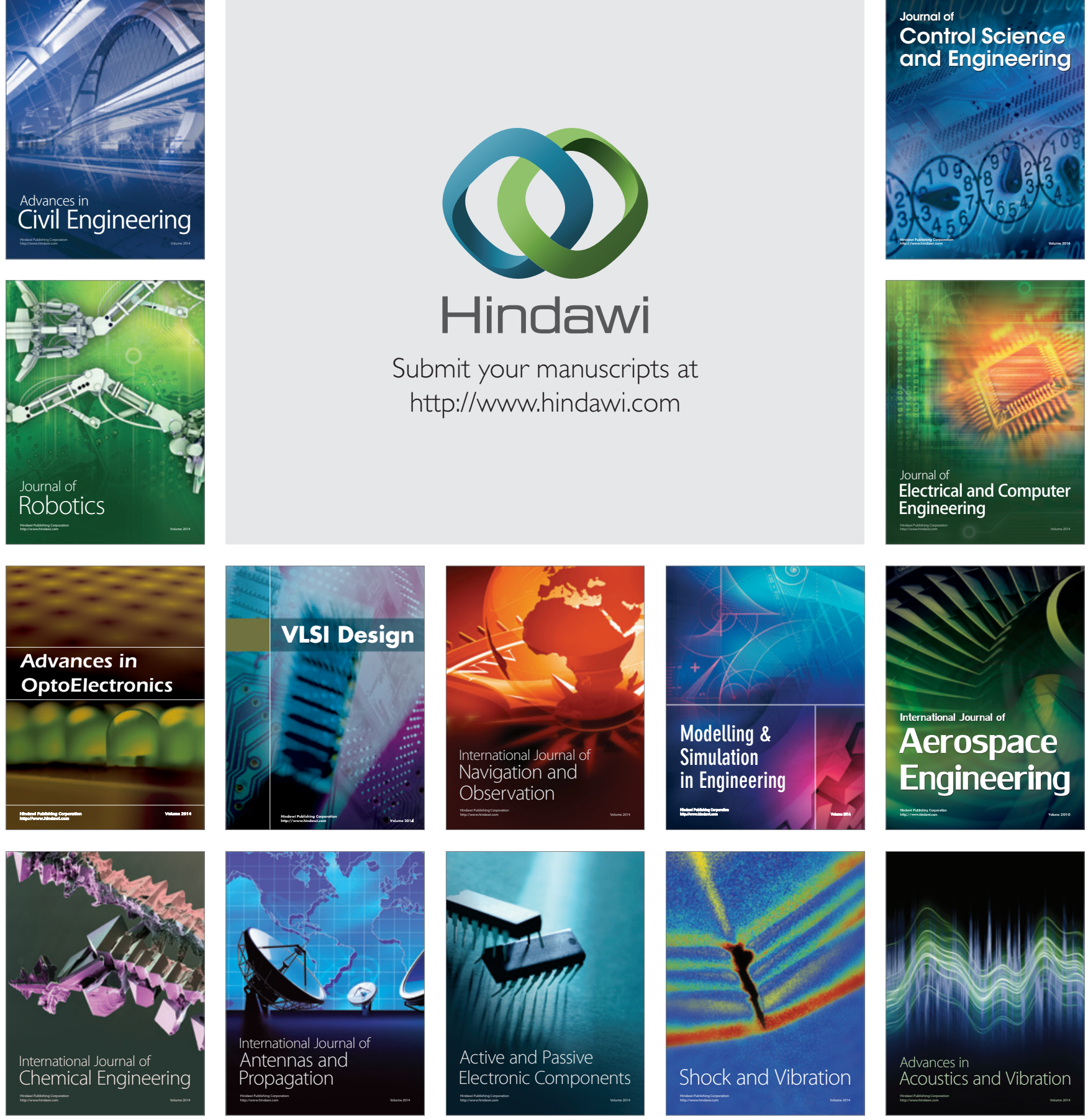